
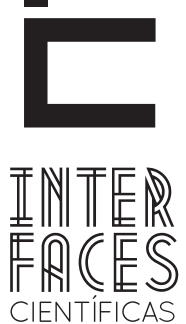

SAÚDE E AMBIENTE

\title{
AS CONSEQUÊNCIAS DA SÍFLLIS CONGÊNITA NO BINÔMIO MATERNO-FETAL: UM ESTUDO DE REVISÃO
}

Brígida Cabral Souza ${ }^{1}$

\section{RESUMO}

Dentre as várias doenças que podem ser adquiridas durante o período gravídico-puerperal, a sífilis é a que possui as maiores taxas de transmissão. Doença multifacetada, quando presente na gestação, pode levar efeitos deletérios, tanto para a mãe como para o feto, caso não seja diagnosticada e tratada o mais precoce possível. 0 objetivo deste trabalho é mostrar as consequências da Sífilis congênita, com a finalidade de sensibilizar os profissionais que prestam assistência a esse público. Trata-se de uma revisão da literatura pertinente, presente nas bases de dados Pubmed, PEDro e Cochrane. Foram selecionados 32 trabalhos, mas apenas 16 artigos apresentaram re-
Licia Santos Santana²

levância para serem discutidos nesta revisão. Após análise dos mesmos, chegou-se à conclusão de que as consequências são inúmeras, podendo levar ao aumento da taxa de transmissão e à morte fetal. Para que isso seja evitado, é necessário um pré-natal mais oportuno, com consultas de melhor qualidade, além da descentralização dos testes rápidos, o que levará ao tratamento mais precoce da infecção.

\section{PALAVRAS- CHAVE}

Sífilis. Gravidez. Transmissão. 


\section{ABSTRACT}

Among the various diseases that can be acquired during pregnant and puerperal cycle, syphilis is the one that has the highest rates of transmission. Multifaceted disease, when present in pregnancy can lead deleterious effects for both the mother and the fetus, if not diagnosed and treated as early as possible. The objective of this work is to show the consequences of congenital syphilis, in order to sensitize the professionals who provide care to the public. This is a review of the relevant literature, present in PubMed, Cochrane and PEDro databases. We selected 32 studies, but only 16 articles had relevance to be discussed in this review. After their analysis, it was concluded that the consequences are numerous and may lead to increased transmission rate and fetal death. To avoid this, you need a more timely prenatal care, best quality and the decentralization of rapid tests, which will lead to earlier treatment of the infection.

\section{KEYWORDS}

Syphilis. Pregnancy. Transmission.

\section{RESUMEN}

Entre las diversas enfermedades que se pueden adquirir durante el embarazo y el puerperio, la sífilis es la que tiene los mayores índices de transmisión. Enfermedad multifactorial, cuando se presenta durante el embarazo puede provocar efectos perjudiciales tanto para la madre como para el feto, si no es diagnosticada y tratada tan pronto como sea posible. El objetivo de este trabajo es mostrar las consecuencias de la sífilis congénita, con el fin de sensibilizar a los profesionales que prestan atención al público. Esta es una revisión de la literatura relevante, presente en la base de datos PubMed, Cochrane y Pedro. Se seleccionaron 32 estudios, pero sólo 16 artículos se mostraron pertinentes para ser discutidos en esta revisión. Después de su análisis, se concluyó que las consecuencias son numerosas y pueden conducir al aumento de la tasa de transmisión y a la muerte fetal. Para evitar esto, es necesario un control prenatal más oportuno y de mejor calidad, y la descentralización de las pruebas rápidas, lo que conducirá a un tratamiento temprano de la infección.

\section{PALABRAS CLAVE}

La Sífilis. El Embarazo. La Transmisión.

\section{INTRODUCC̄̃O}

A sífilis é uma doença infecto-contagiosa, que tem como agente etiológico o Treponema pallidum. Consiste em uma bactéria que é transmitida na relação sexual, transfusão sanguínea, no contato com lesões mucocutâneas e ao feto por via transplacentária, o que configura a sífilis congênita (SC) (MILANEZ e AMARAL, 2008). Dentre as várias doenças que podem ser adquiridas durante o período gravídico-puerperal, a sífilis é a que possui as maiores taxas de transmissão (MINISTÉRIO DA SAÚDE, 2006)
É uma doença multifacetada que, quando presente na gestação, pode levar efeitos deletérios, tanto para mãe como para o feto, caso não seja diagnosticada e tratada, o mais precoce possível. Sua evolução é vista por estágios que se alternam entre sintomáticos e assintomáticos, podendo afetar qualquer órgão do corpo humano. As alterações fisiopatogênicas observadas na gestante são as mesmas que ocorrem na mulher que não se encontra grávida. Quando não tratada, acarreta vários problemas, podendo compreender dis- 
túrbios dermatológicos, neurológicos, ósseos e cardiovasculares (MINISTÉRIO DA SAÚDE, 2006). Uma de suas consequências seria o abortamento espontâneo, morte fetal e neonatal, prematuridade e danos à saúde do recém-nascido com efeitos psicológicos e sociais (RODRIGUES e GUIMARÃES et al., 2004).

O controle da sífilis congênita constitui um dos maiores desafios à saúde pública. A Organização Mundial de Saúde adverte que a eliminação da SC contribuirá para três dos grandes objetivos de desenvolvimento do milênio: a redução da mortalidade infantil, melhoramento da saúde materna e a luta contra HIV/AIDS dentre outras doenças (SILVA et al., 2010).

No Brasil, a luta pelo controle da SC vem ocorrendo há anos. Metas foram estabelecidas pelo Ministério da Saúde, programas de incentivo ao rastreamento da sífilis no pré-natal foram propostos, as campanhas são realizadas, manuais de orientações para profissionais de saúde foram divulgados, a doença foi incluída no registro do SINAN, entretanto, as taxas ainda permanecem elevadas. (SILVA et al., 2010).

De forma individual, Araújo et al. (2006), assinala que dentre os fatores que contribuem para o aumento dos casos de SC, estão a negligência por parte das

\section{METODOLOGIA}

Trata-se de uma revisão da literatura realizada a partir de buscas nas bases de dados Scielo, Pubmed, PEDro, Medline, Bireme, Cochrane no período de dezembro de 2012 a março de 2013, utilizando os seguintes descritores: sífilis congênita, gravidez, doenças sexualmente transmissíveis, Infecções por treponema. Os critérios de seleção foram artigos publicados nos últimos dez anos, incluindo os ensaios clínicos e revisões que falam sobre a sífilis congêni- autoridades de saúde e agentes de saúde na adoção de medidas preventivas, o aumento do número de mães solteiras e adolescentes, a precocidade e promiscuidade sexual, a ausência de conhecimento da população sobre a doença e principalmente, a baixa qualidade do pré-natal no atendimento às gestantes e até mesmo, a falta de acesso a esse serviço.

Essa doença compõe o quadro de causas de morbimortalidade perinatal evitáveis. A ocorrência de SC em mulheres que receberam assistência pré-natal revela que as oportunidades de triagem, diagnóstico e tratamento não estão sendo aproveitadas. É fato que a doença possui um protocolo bem estabelecido e de baixo custo. Sendo assim, as ações direcionadas à eliminação da SC dependem, especialmente, da qualificação na assistência à saúde (MACHADO e CYRINO, 2011).

Tendo em vista o grande número de gestantes com sífilis e a importância da doença nesse contexto, esse trabalho objetiva descrever as consequências da Sífilis congênita em gestantes, com a finalidade de sensibilizar os profissionais que prestam assistência às mesmas. Nossa proposta é popularizar o tema para conscientizar os profissionais de saúde quanto à importância da prevenção, diagnóstico e tratamento adequado.

ta. A partir desse levantamento, foram identificados 32 artigos, mas apenas 16 foram inclusos. Os artigos experimentais, de revisão e estudo de casos foram apresentados de forma descritiva e com suporte para a discussão. 


\section{RESULTADOS}

Constatamos, em nossa pesquisa na literatura, uma atenção maior dos pesquisadores quanto ao aumento da incidência, a deficiência na assistência pré-natal e ao conhecimento de puérperas acometidas pela sífilis, o que é perfeitamente compreensivo, dado à gravidade dessa doença. Neste sentido, percebemos a importância de investigar outra vertente, que também poderá ser utilizada em futuras ações de prevenção, diagnóstico e tratamento. Apesar da escassez de artigos diretamente relacionados ao nosso objetivo, foi possível estudar os efeitos deletérios no binômio materno-infantil.

Ao término da busca, foram localizados 32 trabalhos com os descritores mencionados. Levando em consideração os critérios de seleção dessa revisão, foram incluídos 16 artigos.

\section{DISCUSSÃO}

A sífilis tem um alto poder de transmissão. Além disso, o embrião pode ser infectado em qualquer fase gestacional ou estágio da doença materna. 0 risco de transmissão vertical da sífilis varia de $30 \%$ a $100 \%$, dependendo do estágio da doença e a duração de exposição do feto intra-útero. De acordo com o Ministério da Saúde, 2007, a taxa de transmissão vertical da sífilis em mulheres não tratadas é de 70 a 100\% nas fases primária e secundária da doença, reduzindo para aproximadamente $30 \%$, nas fases tardias da infecção materna (MILANEZ e AMARAL, 2008; ARAUJO et al., 2006; MAGALHAES et al., 2011).

De acordo com a pesquisa de Machado e Cyrino (2011), afirma-se que a sífilis congênita pode se manifestar por meio de abortamentos, natimortos, morte perinatal ou pode manter-se sob forma assintomática em recém-nascidos que poderão apresentar sinais e sintomas em fases posteriores da vida. Além dos efeitos deletérios, ocorridos no concepto, a genitora também sofre alterações biopsicossociais.

Dentre os estudos pesquisados, vários autores destacaram que a sífilis é uma causa de grande morbidade na vida intra-uterina, levando a desfechos negativos na gestação. Afirmam que aproximadamente
$40 \%$ dos conceptos infectados evoluem para aborto espontâneo (perda gestacional, ocorrida antes de 22 semanas de gestação, ou com peso menor a 500 gramas), natimorto (após 22 semanas de gestação ou com peso igual ou maior a 500 gramas) ou morte Perinatal (RODRIGUES e GUIMARÃES et al., 2004; MINISTÉRIO DA SAÚDE, 2007; ARAUJO et al., 2006; SARACENI et al., 2005; MESQUITA et al., 2012; SANTOS e ANJOS, 2009; MAGALHAES et al., 2011; MINISTÉRIO DA SAÚDE, 2006; MILANEZ e AMARAL, 2008).

Em 2005, Saraceni et al. analisou 7.309 óbitos perinatais (4.215 fetais e 3.094 neonatais precoces) de filhos de mães residentes no Município do Rio de Janeiro, no período de 1999 a 2002. Destes óbitos fetais, $292(4,0 \%)$ tiveram como causa básica a sífilis congênita, sendo 222 (5,3\%) fetais e 70 (2,3\%) neonatais precoces. Os autores afirmam que a sífilis materna já foi associada a um risco dez vezes maior de ocorrência de perda fetal. No estudo realizado por Sá et al. (2001) na Maternidade Escola da Universidade Federal do Rio de Janeiro, o resultado foi um total de 749 casos notificados de sífilis congênita; destes, 82 (11\%) compunham-se de óbitos fetais e 42 (5,7\%) de óbitos neonatais em 1998. Já de acordo com a entrevista realizada por Silva et al. (2010) em sua pesqui- 
sa, das onze mulheres estudadas, duas vivenciaram a morte perinatal dos seus conceptos, como demonstra o relato de uma das pacientes: "O meu filho que teve sífilis foi o que morreu... Ele (criança) nasceu vivo, cego e sem conseguir se mexer e depois de dois dias ele morreu... Eu não fiz pré-natal por que eu não gosto, eu acho muito chato".

Diante desses achados, fica comprovado que a mortalidade perinatal realmente possui forte relação com a sífilis congênita. Vale ressaltar que o coeficiente de mortalidade infantil é um indicador de saúde, que além de informar a respeito dos níveis de saúde de uma população, sintetiza as condições de bem-estar social, político e ético. Lembramos ainda que a sífilis congênita se insere no quadro de causa perinatal evitável, pois é possível fazer o diagnóstico e proceder ao tratamento efetivo na gestação.

Ao nascerem filhos de mães com sífilis tratada ou não de forma inadequada, estes podem se apresentar sintomáticos ou assintomáticos. Entretanto, mais de $50 \%$ dos casos notificados são assintomáticos ao nascimento, o que dificulta o diagnóstico logo após o nascimento. Como o surgimento dos primeiros sintomas, geralmente acontece nos primeiros três meses de vida, temos frequentemente diagnóstico tardio, o que acarreta em sérias consequências para a criança (RODRIGUES e GUIMARÃES et al., 2004; MINISTÉRIO DA SAÚDE, 2007; ALVARES e MEZZACAPPA e POTERIO, 2002; MACHADO E CYRINO, 2011; MINISTÉRIO DA SAÚDE, 2006; SECRETARIA DO ESTADO DE SP, 2008).

A manifestação da sífilis congênita é dividida em precoce (antes do $2^{\circ}$ ano de vida) ou tardia (a partir do $2^{\circ}$ ano de vida), sendo a última a que acarreta maiores prejuízos à sua saúde. Dentre as manifestações clínicas mais frequentemente citadas entre os pesquisadores na sífilis congênita precoce, destaca-se a prematuridade (nascimento antes de 37 semanas de idade gestacional) e o baixo peso ao nascer (inferior a 2.500g) (MACHADO E CYRINO, 2011; MINISTÉRIO DA SAÚDE, 2006; SECRETARIA DO ESTADO DE SP, 2008).
Reafirmando essa informação, Campos et al. (2010), em seu estudo realizado em cinco maternidades públicas localizadas no Município de Fortaleza-Ceará, investigou uma amostra de 58 gestantes com VDRL reagente e observou o resultado de prematuridade em $36,2 \%$ dos casos estudados.

Complementando a afirmação acima, o estudo realizado por Saraceni et al. (2005), citado anteriormente, apresenta-nos como resultado, a prematuridade e o baixo peso ao nascer maiores entre os óbitos perinatais por sífilis congênita do que por outras enfermidades. 0 baixo peso ao nascer nessa pesquisa ocorreu em $95,5 \%$ dos casos, e a prematuridade, em $90,5 \%$. Sendo assim, rememoramos que a sífilis é uma das causas de prematuridade em crianças e baixo peso ao nascer, consequentemente aumentando o risco da mortalidade perinatal.

Sabe-se que a sífilis congênita pode afetar todos os órgãos do corpo humano. Sendo assim, encontram-se manifestações clínicas em quase todos os sistemas no concepto. Entre elas, estão as lesões cutâneo-mucosas, lesões ósseas, lesões do sistema nervoso central, lesões do aparelho respiratório, dentre outras.

Alguns dessas alterações são demonstradas no estudo de caso realizado por Alvares, Mezzacappa e Poterio (2002), no qual a criança nasceu pré-termo, com peso 1.910 gramas e idade gestacional de 34 semanas, no Centro de Atenção Integral à Saúde da MuIher (Caism)/Unicamp. A gestante apresentou exame VDRL positivo (1/32) durante a investigação pré-natal, sem tratamento relatado. 0 recém- nascido apresentou lesões de pele bolhosas em palmas das mãos e plantas dos pés, e lesões eritematosas disseminadas, características da doença, e também com discreta hepatomegalia e monoparesia do membro superior esquerdo. O VDRL no RN foi de 1/256 e TPHA reagente. 0 exame do líquor cefalorraquiano demonstrou hiperproteinorraquia (229 mg/dl) e VDRL 1/2, caracterizando neurolues. Exames radiológicos dos ossos longos, 
com 24 horas de vida, demonstraram lesões metafisárias nos membros inferiores e superiores, compatíveis com a doença. Com 15 dias de vida, novas radiografias constataram espessamento periosteal nas diáfises do úmero esquerdo, fêmur e tíbia bilateralmente, bem como exuberantes calos ósseos nas extremidades proximais dos fêmures, tíbia direita e em ambas as extremidades do úmero esquerdo. Com quatro meses de vida, o controle radiológico evidenciou remodelamento ósseo metafisário, com desaparecimento dos calos ósseos nos membros superiores e inferiores, persistindo espessamento periosteal nos fêmures.

De acordo com Magalhães et al. (2011), em seu estudo, afirma que existem duas possibilidades para a ocorrência da SC. A primeira, a mulher é infectada antes da gestação, e na segunda, a paciente é contaminada durante a gestação. Assim, o espectro de desfechos possíveis torna-se variado, de acordo com o estágio gestacional e da infecção, que pode ainda ter um terceiro determinante no desenvolvimento do sistema imunológico fetal. Segundo o Ministério da Saúde (2006), as alterações fisiopatogênicas ocorridas na gestante acometida pela sífilis, são as mesmas encontradas em uma mulher não gestante (MAGALHÃES et al., 2011)

Quando a gestante adquire sífilis durante o período da formação fetal, ela inicia pelo primeiro estágio, denominado sífilis primária, que se caracteriza pelo aparecimento do cancro duro, uma lesão erosada ou cerada, geralmente única, indolor, com bordas endurecidas, fundo liso e brilhante no local de inoculação do agente (MINISTÉRIO DA SAÚDE 2006, MINANEZ E AMARAL 2008, MACHADO E CYRINO 2011, MS 2007, SANTOS E ANJOS 2009). Geralmente aparece nos pequenos lábios, paredes vaginais e colo uterino, podendo passar despercebida. A lesão aparece entre 10 e 90 dias (média de 21), após o contato sexual infectante, juntamente com aumento os linfonodos locais (Adenopatia regional não supurativa, móvel, indolor e múltipla) (MINISTÉRIO DA SAÚDE, 2007; SANTOS E ANJOS, 2009; MINANEZ E AMARAL, 2008).
Na fase secundária, tanto a gestante que já possuía a sífilis como a que adquiriu pode transmitir, uma vez que as lesões se manifestam em média oito semanas após o desaparecimento espontâneo do cancro. A apresentação mais comum da fase secundária são as máculas. Entretanto, as lesões podem assumir diversos aspectos e dificultar o diagnóstico (MINISTÉRIO DA SAÚDE, 2007; MILANEZ E AMARAL, 2008; MACHADO E CYRINO, 2011).

No relato de caso realizado por Reis et al. (2007), foi estudada uma gestante, com 28 anos de idade, multípara com quatro gestações, um aborto espontâneo há oito anos, sem parceiro fixo. Não realizou pré-natal, não sabe a última menstruação, tabagista, usuária de crack, três parceiros sexuais nos últimos 12 meses, sendo o último há um mês, o qual apresentava secreção peniana purulenta. Procurou assistência em decorrência da presença de lesões em vulva, iniciadas há 15 dias, associadas à febre não-aferida. Ao exame físico geral, foram evidenciados linfonodos inguinais palpáveis e indolores bilateralmente e pápulas eritematosas em abdome e dorso. Ao exame da genitália externa, observou-se intenso edema de vulva, lesões papulosas, indolores, com superfície lisa, em regiões perineal e vulvar, características de sifílides.

Os sintomas das fases, primária e secundária, regridem espontaneamente, mesmo sem tratamento, e as lesões da fase terciária, que surgem após décadas são, na atualidade, raramente descritas. Sendo assim, essa fase acontece geralmente quando a gestante já possui a sífilis antes da gestação (MILANEZ e AMARAL, 2008)

A sífilis terciária ou latente ocorre após a secundária e se subdivide em Sífilis recente, que é uma fase de duração variável, em que não se observam sinais e sintomas clínicos. O diagnóstico é realizado exclusivamente através de testes sorológicos. E a Sífilis tardia, onde os sinais e sintomas surgem em um período variável após 3 a 12 anos, ou mais, do contágio. Suas 
manifestações clínicas mais comuns são as lesões cutâneo-mucosas, que se apresentam como tubérculos ou gomas; apresentação neurológica, tal como o tabes dorsalis e demência; doença cardiovascular sob a forma de aneurisma aórtico; comprometimento articular (artropatia de Charcot) (SANTOS e ANJOS, 2009; MINISTÉRIO DA SAÚDE, 2007; MINISTÉRIO DA SAÚDE, 2006; MACHADO e CYRINO, 2011)

Além das alterações fisiopatogênicas observadas na gestante, a sífilis também causa efeitos psicológicos e sociais na vida dessa mulher (Machado e Cyrino, 2010). Estudos apontam para o fato de que mães, que já perderam outros filhos por sífilis congênita, apresentam um maior grau de sentimento de culpa. No estudo realizado por Victor et al. (2010), participaram 20 puérperas que estavam acompanhando seus recém-nascidos para tratamento de sífilis congênita em uma maternidade de referência em Fortaleza-CE, entre fevereiro/junho/2008. Os achados revelaram sentimentos distintos, algumas puérperas demonstraram tristeza e sofrimento pelos seus filhos na hora da realização da medicação, outras puérperas se culpam pelo sofrimento do filho.

Já na pesquisa de Silva e outros (2010), um estudo descritivo, de natureza qualitativa, que utilizou como instrumento de coleta de dados, a entrevista semiestruturada, na qual foram avaliadas 11 mulheres, encontramos nos relatos das mesmas, o mal estar e a tristeza, após a constatação da doença. Elas manifestaram sentimentos e expressões de temor, desespero e impotência diante do internamento hospitalar e dos procedimentos aos quais seus filhos tiveram que ser submetidos. Além disso, ao serem comunicadas sobre sua infecção e a de seu filho, relataram sentimentos de solidão e desprezo.

O estudo de Machado e Cyrino (2011) mostra que os pais/famílias de crianças com anomalia congênita experimentam diferentes sentimentos. A princípio há o choque da descoberta de que não foi gerada uma criança saudável e que corresponda ao esperado pela sociedade, seguido do desencadeamento de sentimentos de culpa e tristeza. Em alguns casos, é desenvolvido um sentimento de superproteção: os pais passam a esconder sua criança portadora de anomalia, buscando protegê-la dos olhares maldosos e da perversidade da sociedade. Alguns já se conformam com a situação quando acreditam que ter um filho com deficiência é a vontade de Deus.

De acordo com o estudo de Mesquita et al (2012), os gastos hospitalares com o tratamento da SC ultrapassam os gastos que se teriam com a efetiva profilaxia da SC, se houvesse um programa de assistência pré-natal de qualidade, mostrando que devesse fazer mais campanhas educativas, programa de incentivo ao uso de proteção na relação sexual em mulheres, principalmente aquelas que não têm um parceiro fixo, durante todo o seu ciclo de vida e incentivar a procurarem o médico especialista na área com mais frequência, para que assim, o diagnóstico fosse precoce e consequentemente, os gastos hospitalares fossem diminuídos.

\section{CONCLUSÃO}

Apesar da necessidade de mais análises científicas sobre o tema abordado, os estudos realizados e incluídos nesta revisão evidenciam os efeitos deletérios da sífilis congênita no binômio materno infantil. Com

esta revisão, observamos que para atingimos a erradicação, é necessário um pré-natal mais oportuno, com consultas de melhor qualidade, além da descentralização dos testes rápidos, o que levará ao tratamento 
mais precoce da infecção, para que assim, diminua a mortalidade fetal. Acreditamos também que sensibilizaremos os profissionais que prestam assistência à saúde da gestante com sífilis, para que eles reflitam sobre o motivo da reincidência de casos de sífilis congênita.

\section{REFERÊNCIA}

ALVARES, B. R.; MEZZACAPPA, M. A. M. S.; POTERIO, C. B. Sífilis congênita simulando a síndrome da criança espancada - relato de caso. Campinas, SP, 2002

ARAUJO, E. C. et al. Importância do pré-natal na prevenção da sífilis congênita. Revista Paraense de Medicina, v: 20.Pará, 2006.

BRASIL. Ministério da Saúde. Diretrizes para controle da Sífilis Congênita. Secretaria Nacional de Vigilância em Saúde. Brasília, DF, 2006.

BRASIL. Ministério da Saúde. Protocolo para a prevenção de transmissão vertical de HIV e sífilis. Brasília, DF, 2007.

CAMPOS, A. L. A. et al. Epidemiologia da sífilis gestacional em Fortaleza, Ceará, Brasil: um agravo sem controle. Cad. Saúde Pública, Rio de Janeiro, 26(9): 1747-1755, set, 2010

MACHADO, F. M. e CYRINO, R. M. F. Planejamento do enfermeiro na inserção social de crianças portadoras de sífilis congênita. Faculdade Católica Salesiana do Espírito Santo, Vitoria, 2011.

MAGALHÃES, D. M. DOS S. et al. A sífilis na gestação e sua influência na morbimortalidade materno-infantil. Brasília/DF, 2011.

MESQUITA, K. O. et al. Análise dos Casos de Sífilis Congênita em Sobral, Ceará: Contribuições para Assistência Pré-Natal. J bras Doenças Sex Transm, 2012.

MILANEZ, H.; AMARAL, E. Por que ainda não conseguimos controlar o problema da sífilis em gestantes e recém-nascidos? Campinas/SP. Revista Brasileira de Ginecologia Obstetrícia. 30(7): 325-7, p. 325, 2008.

REIS, H. L. B. Sífilis recente em gestante e efeito prozona na sorologia: relato de caso. J bras Doenças Sex Transm, 2007.

RODRIGUES, C. S.; GUIMARÃES, M. D. C. E GRUPO NACIONAL DE ESTUDO SOBRE SÍFILIS CONGÊNITA. Positividade para sífilis em puérperas: ainda um desafio para o Brasil. Rev Panam Salud Publica. 2004; 16(3):168-75.

SANTOS, V. C.; ANJOS, K. F. Sífilis: uma realidade prevenível. Sua erradicação, um desafio atual. Revista Saúde 
e Pesquisa, v. 2, n. 2, p. 257-263, 2009.

SARACENI, V. ET AL. Mortalidade perinatal por sífilis congênita: indicador da qualidade da atenção à mulher e à criança. Cad. Saúde Pública, Rio de Janeiro, 2005.

SILVA, M. R. F. da et al. Percepção de mulheres com relação à ocorrência de sífilis congênita em seus conceptos. Rev. APS, Juiz de Fora, v. 13, n. 3, p.301-309, 2010. Disponível em: <http://www.aps.ufjf.br/index.php/aps/ article/view/722/341>. Acesso em: 3 jan 2013.

SÃO PAULO. Secretaria de Estado da Saúde. Sífilis congênita e sífilis na gestação. São Paulo, 2008.

VÍCTOR, J et al. Sífilis congênita: conhecimento de puérperas e sentimentos em relação ao tratamento dos seus filhos. Rev. Eletr. Enf. [Internet]. 2010. Disponível em; <http://www.fen.ufg.br/revista/v12/n1/v12n1a14. htm>. Acesso em: 3 jan. 2013. 